\title{
Animal experiments under fire for poor design
}

In the contentious world of animal research, one question surfaces time and again: how useful are animal experiments as a way to prepare for trials of medical treatments in humans? The issue is crucial, as public opinion is behind animal research only if it helps develop better drugs. Consequently, scientists defending animal experiments insist they are essential for safe clinical trials, whereas animal-rights activists vehemently maintain that they are useless.

Now a British team has made the first attempt to answer the question in a scientific way, and the result suggests that animal researchers need to raise their game. The team claims that animal experiments are often poorly designed, and so fail to lay the ground properly for subsequent human studies.

The study looked at six treatments that have been evaluated in detail in human trials. The researchers assessed whether animal studies had accurately predicted the outcome of the human work, a task that involved reviewing more than 200 papers. In three of the six cases, the answer was no (P. Perel et al. Br. Med. J. doi:10.1136/bmj.39048.407928.BE; 2006).

Ian Roberts, an epidemiologist at the London School of Hygiene and Tropical Medicine and one of the study's authors, says further analysis revealed that poor-quality methodologies and inappropriate models caused the failures. Some studies didn't randomize the allocation of animals to control and treatment groups properly, whereas others showed evidence for publication bias - the tendency not to publish negative results. A third set involved inappropriate models: for example in studies of head injury, treatments were administered just five minutes after rodents were deliberately injured, but humans are typically treated within three hours of accidents.

Roberts would like to see animal researchers learn from clinical trials, where similar problems are now being addressed. He says animal researchers should register experiments before they begin, for example, to ensure the results are reported and to avoid publication bias. He would also like more detailed reporting of experimental procedures, so reviewers can ignore low-quality and potentially biased work. "All the things that happen in clinical trials need

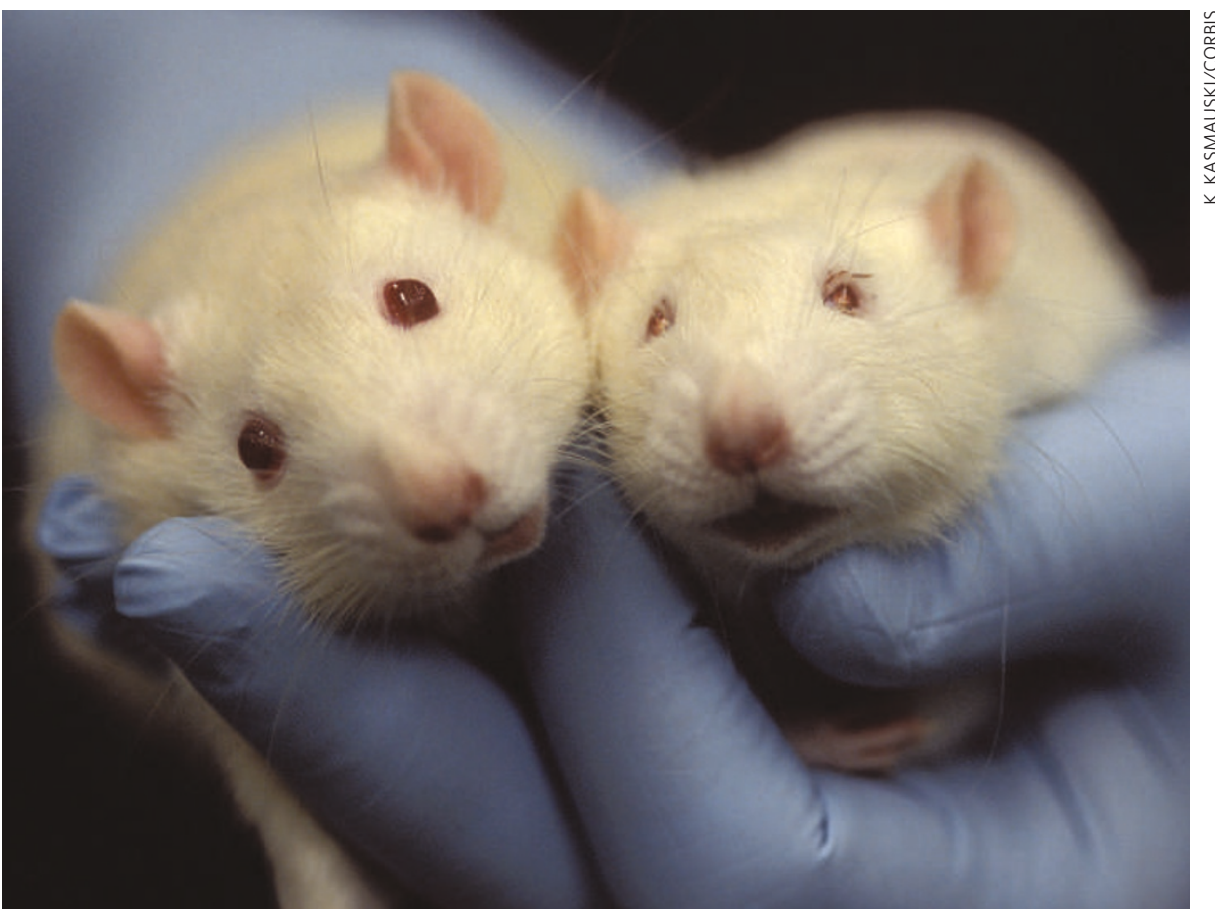

Are animals being wasted in badly thought through experiments?

to happen in animal experiments," he says.

Some scientists familiar with both animal and human studies have welcomed the paper, saying it highlights the need for researchers from the two arenas to work more closely on the design of animal experiments. But others have questioned whether a crude comparison of trials says anything meaningful about the usefulness of animal work.

In many cases, say critics, animal studies are designed to address specific questions not related to a drug's effectiveness, such as how it distributes around the body, and so cannot be compared to clinical trials. Others point out that applying the standards used in human trials to animal experiments could be prohibitively expensive. For example, initial tests are often run by small groups of researchers. With just one or two scientists, it is impractical to properly blind a study. It is also worth remembering that researchers apply an "intelligent filter" to animal results when designing clinical trials, says Robert Lechler, an immunologist at Kings
College London, and don't expect the results to map straight over to human work.

Roberts counters that his paper looked only at animal studies that were specifically designed to model human disease. And he adds that despite the low cost, small-scale studies are pointless if they do not produce results that people can have confidence in, even if researchers are aware of the limitations. "If you have something cheap and cheerful you have a high probability that the result is wrong," he says. "Just think of the money that is wasted down the line if you follow up that lead."

The results are likely to feature in future campaigns by animal-rights groups as evidence that animal research doesn't benefit medicine. But the authors stress that this would be an error, and that a comparison of just six treatments cannot be used to justify general statements about animal research. "It's not a polemic against animal researchers," says Peter Sandercock, a neurologist at the University of Edinburgh and another author on the study. "But we need to be aware that there are biases in the animal trials."

Jim Giles 